\title{
Gemini/GMOS detection of stellar velocity variations in the ionising cluster of 30 Dor
}

\author{
Guillermo Bosch ${ }^{1}$, Elena Terlevich ${ }^{2}$ and Roberto Terlevich ${ }^{2}$ \\ ${ }^{1}$ Facultad de Ciencias Astronómicas y Geofísicas \& IALP Paseo del Bosque s/n, \\ 1900 La Plata, Argentina \\ email:guille@fcaglp.unlp.edu.ar \\ ${ }^{2}$ INAOE, Tonantzintla, Apdo. Postal 51, 72000 Puebla, México
}

\begin{abstract}
We have analysed spectra obtained with the Gemini Multi Object Spectrograph (GMOS) for more than 50 stars in the ionising cluster of 30 Doradus during a seven epochs observing campaign at Gemini South. We derive a binary candidate rate of about $50 \%$, which is however consistent with an intrinsic $100 \%$ binary rate among massive stars. After decontaminating the sample from the stars that show binary orbital motions, we were able to calculate the "true" cluster velocity dispersion and found it to be about $8 \mathrm{~km} \mathrm{~s}^{-1}$. This value implies a virial mass of about $4.5 \times 10^{5} \mathrm{M}_{\odot}$ which is consistent with previous photometric mass determinations therefore suggesting that NGC 2070 is a firm candidate for a future globular cluster.
\end{abstract}

Keywords. stars: early-type, stars: kinematics, binaries: spectroscopic, galaxies: individual (LMC), Magellanic Clouds

\section{Introduction}

30 Doradus in the LMC is the nearest available example of a young and massive starburst cluster. Given its proximity it is possible to perform a highly detailed study of its stellar component. The large number of massive stars present in this single cluster allows the statistical analysis of several parameters at a level of significance that is not available in the local and smaller Galactic clusters.

When analysing the stellar kinematics of the 30-Dor ionising cluster, Bosch (1999) and collaborators pointed out that the observed radial velocity dispersion of stars within the cluster, can be strongly affected by the orbital motions of massive binaries. Bosch et al. (2001) performed a radial velocity analysis on the stars that conform the ionising cluster of 30 Dor. They suggested that the very large value of the velocity dispersion $(\sigma \sim 35 \mathrm{~km}$ $\mathrm{s}^{-1}$ ) obtained for the OB stars within NGC 2070, the ionizing cluster in 30 Dor, could be due to the presence of an underlying binary population. From Montecarlo simulations Bosch \& Meza (2001) estimated that this contribution could be as large as $36 \mathrm{~km} \mathrm{~s}^{-1}$, provided all stars in the cluster belonged to a binary pair. However, it is necessary to confirm this assumption with direct evidence. The issue of binary frequency is still subject to debate, although there is a relatively high number of spectroscopic and visual binaries reported in the literature (Lada 2006).

Here we present a new set of observations of NGC 2070 obtained with the Gemini Multi Object Spectrograph (GMOS) at Gemini South. These comprise multi object optical spectroscopy of 50 early-type stars observed at least in six different epochs. The aim is to detect spectroscopic binary stars from variations in their radial velocities. 
Table 1. Log of observations. Observing dates are listed in column 1 and Heliocentric Julian dates for masks I and II are shown in columns 2 and 3 respectively.

\begin{tabular}{lrr}
\hline & Mask I & Mask II \\
\hline 07 Sep. 2005 & 2453620.89 & \\
19 Dec. 2005 & & 2453723.58 \\
20 Dec. 2005 & 2453724.58 & \\
22 Dec. 2005 & & 2453726.68 \\
23 Dec. 2005 & 2453727.65 & \\
24 Dec. 2005 & & 2453728.59 \\
29 Nov. 2006 & 2454068.73 & \\
30 Nov. 2006 & & 2454069.70 \\
24 Dec. 2006 & 2454093.83 & \\
31 Dec. 2006 & & 2454100.86 \\
01 Jan. 2007 & 2454101.79 & 2454101.73 \\
09 Jan. 2007 & & 2454109.66 \\
\hline
\end{tabular}

\section{Observations and data reduction}

Observations were performed at Gemini South Observatory (Proposals GS-2005B-Q-2 and GS-2006B-Q-21) using two multislit masks. Targets within each mask were selected from a previous imaging run with GMOS according to their spectral types as determined and compiled in Bosch et al. (1999).

Spectra were obtained during nights of September and December 2005 for the first run and December 2006 and January 2007 for the second run. Approximate HJD for the listed observations are shown in table 1 . The instrument was set up with the B1200 grating $R \sim 3700$ centred at about $4500 \AA$ which yields a resolution of $0.25 \AA$ per pixel at the CCD. Although the wavelength range varies slightly in MOS spectroscopy, the region from $3900 \AA$ to $5500 \AA$ is covered by our spectra. The total integration time was split in three to allow for the wavelength dithering pattern needed to cover the gaps between GMOS CCDs. Overall signal to noise ratios are above 150. These ratios are measured on reduced spectra, dividing the average value of the stellar continuum by the scatter over the same spectral range.

\section{Radial velocities}

Radial velocities were derived measuring absorption line profiles with the aid of the ngaussfit task within the STSDAS/IRAF package, following a similar procedure as the one described in Bosch et al. (2001). This allowed us to derive individual radial velocities for each spectral line, and handle each element (or each ion in the case of He) separately. Stellar radial velocities were derived using the best set of lines available, according to the star's spectral type. Although this means we are not using the same set of lines for the whole sample, we strictly kept the same set of lines for the same star on different epochs when looking for radial velocity variations. Uncertainties introduced when fitting individual Gaussians to the absorption profiles are of the order of $5 \mathrm{~km} \mathrm{~s}^{-1}$.

Nebular spectra were wavelength calibrated together with the stellar spectra, to provide a strong template to check for variations of our radial velocities zero-point at different epochs. Nebular emission lines have strong narrow profiles, enabling us to cross-correlate nebular spectra obtained on different nights, using fxcor within IRAF, that yields accurate determinations of radial velocity differences, if present. Radial velocities derived for each night $\left(V_{\text {neb }}\right)$ were very stable when observations on different epochs are compared and their variations $\left|\Delta V_{\text {neb }}\right|$ were found to be negligible $\left(\left|\Delta V_{\text {neb }}\right| \leqslant 1.0 \mathrm{~km} \mathrm{~s}^{-1}\right.$, $\sigma_{\text {neb }}=3.1 \mathrm{~km} \mathrm{~s}^{-1}$ ) which suggests that there is no systematic shift introducing spurious variations of stellar radial velocities between epochs. 
Table 2. Stellar identifications by Parker (1993) are in column 1. Columns 2 through 15 include radial velocities and uncertainties (all in $\mathrm{km} \mathrm{s}^{-1}$ ) for each epoch of observation. Column 16 lists the ratio between epoch-to-epoch variations and the average uncertainties.

\begin{tabular}{|c|c|c|c|c|c|c|c|c|c|c|c|c|c|c|c|}
\hline Id & $V_{\mathrm{r}}$ & $\sigma_{\mathrm{I}}$ & $V_{\mathrm{r}}$ & $\sigma_{\mathrm{I}}$ & $V_{\mathrm{r}}$ & $\sigma_{\mathrm{I}}$ & $V_{\mathrm{r}}$ & $\sigma_{\mathrm{I}}$ & $V_{\mathrm{r}}$ & $\sigma_{\mathrm{I}}$ & $V_{\mathrm{r}}$ & $\sigma_{1}$ & $V_{\mathrm{r}}$ & $\sigma_{\mathrm{I}}$ & $\frac{\sigma_{\mathrm{E}}}{\left\langle\sigma_{\mathrm{I}}\right\rangle}$ \\
\hline $15(\mathrm{I})$ & 99.1 & 5.5 & & & 272.7 & U & 5 & 4.9 & 2 & 7 & 2 & 6 & & & 9 \\
\hline $32(\mathrm{II})$ & 33.3 & 6.3 & 271.6 & 2 & & 4.8 & 71.3 & 4.3 & 8.3 & 3 & & & & & 1.6 \\
\hline $124(\mathrm{I})$ & 36.0 & 4.2 & 239.3 & 3.7 & 9.7 & 6.0 & 88.7 & 3.3 & 243.5 & 5 & 261.0 & & & & 1 \\
\hline 171(I) & 2.7 & 3.7 & & 4 & & 5.0 & & 4.7 & 276.7 & & 269.2 & 1 & & & .1 \\
\hline 260(II) & 6.0 & & & 9 & & 9.4 & 6.6 & 4.2 & & & 271.5 & 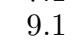 & 3.4 & & 3 \\
\hline 305(II) & 9.5 & 7 & 269.5 & 1 & 9.9 & 6.4 & 55.4 & 3.3 & 258.9 & 8 & 253.1 & 9 & 282.5 & & 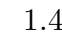 \\
\hline $316(\mathrm{I})$ & 36.7 & 5.0 & 366.0 & 6.7 & 2.8 & 3.9 & 14.5 & 4.1 & 349.8 & 7 & 302.5 & 5.7 & & & 9.4 \\
\hline 485(II) & 77.3 & 5.3 & 260.1 & 5.5 & 55.7 & 8.1 & 278.7 & 3.5 & 251.2 & 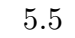 & 259.8 & 6.2 & 2 & 4.0 & 2.0 \\
\hline 531(II) & 9.3 & 7.1 & 247.6 & 3.7 & 21.2 & 9.3 & 60.9 & 3.7 & 276.9 & 9.0 & 324.6 & 11.3 & 241.7 & & .3 \\
\hline (I) & 9.2 & 6.2 & 276.0 & 0 & 72.4 & 3.4 & 232.8 & 3.9 & 358.5 & & 232.1 & 3.9 & & & .4 \\
\hline (II) & 6.2 & & 6 & 8 & .9 & 9.1 & 57.6 & 3.8 & 264.5 & & 6.9 & 5 & 2 & & 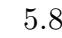 \\
\hline (II) & 32.6 & & 3 & 5 & 0.2 & 6.4 & 76.9 & 3.7 & 281.5 & & 285.2 & 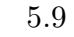 & & & .5 \\
\hline 684(II) & 75.7 & .9 & 4 & 4 & 17.8 & 7.8 & 52.7 & 3. & 254.8 & 0 & 272.5 & 8.7 & 270.7 & 6 & .9 \\
\hline $713(\mathrm{I})$ & 38.3 & 4.7 & 316.1 & 9 & .8 & 4.4 & 20.4 & 5. & 297.0 & 7.2 & 312.6 & 10.9 & & & 6 \\
\hline 716(II) & 74.0 & 3.6 & 273.8 & 7.4 & 66.1 & 4.6 & 271.4 & 3.8 & 275.3 & & 279.8 & 4.1 & 277.5 & 0 & .0 \\
\hline (I) & 32.4 & & 9 & 6 & 6.5 & 5.0 & 31.0 & 4.0 & 248.3 & & 306.0 & .5 & & & 10.1 \\
\hline II) & 3.8 & & & & 0 & 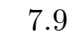 & 3 & & 2 & & .6 & 7 & & & 1.2 \\
\hline (II) & 5.2 & & 2 & 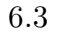 & 7 & 8 & .3 & 3. & 28 & & 1 & & & & .2 \\
\hline & 6.1 & & & 1 & 3 & 7.6 & 8 & 3 & & & & & 2 & & .5 \\
\hline $905(\mathrm{I})$ & 8.5 & 6.9 & 267.4 & 2 & 71.2 & 11.1 & .5 & 4.1 & & 5.1 & & .8 & & & 0.7 \\
\hline $956(\mathrm{I})$ & 68.4 & 6 & 284.5 & 4 & 239.1 & 3.7 & 55.7 & 6. & 270.3 & 71 & 274.3 & .8 & & & .5 \\
\hline $975(\mathrm{II})$ & 7.4 & & & & .7 & 5 & & 3. & 289.2 & & & .0 & 287.2 & 3.8 & .9 \\
\hline (I) & 4.3 & & & & 1 & ) & .0 & 5 & .4 & & .5 & 6 & & & .3 \\
\hline II) & 37.8 & 1.2 & 2 & & $r$ & & .7 & 4. & 27 & & & 4 & & & .3 \\
\hline & 99.5 & ; & 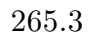 & 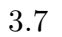 & 5.9 & 8. & 6 & 4. & 2 & 4 & 2 & .2 & 266.4 & & .0 \\
\hline $9(\mathrm{I})$ & 4.2 & & & 6 & & 4.5 & .5 & 0 & .0 & 4.4 & & 1 & & & 3.0 \\
\hline $39(\mathrm{I})$ & 6.3 & & 266.2 & 0 & 9.1 & 4.1 & 59.4 & 4.2 & 257.1 & 5.5 & 271.7 & 4 & & & 1.2 \\
\hline & 3.6 & & & & & 8 & & 8 & 266.5 & 11.9 & 260.9 & 13.0 & & & .6 \\
\hline & .1 & & & & & & & 3 & & & & 10.3 & 294. & & 1.7 \\
\hline & 7.8 & & 2 & 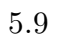 & .2 & 3 & 4 & 6. & .3 & & .9 & 4.6 & & & 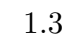 \\
\hline (I) & 8.7 & 8 & 263 & 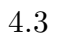 & 8.1 & 15.3 & 5 & 9 . & 26 & 8 & .3 & & & & .5 \\
\hline (I) & 9.0 & & O & 1 & 3 & & & 4 & & & & 9 & & & 1.1 \\
\hline $9(\mathrm{I})$ & 52.7 & & 271.8 & 2 & 5.0 & 6.3 & 1.8 & 4.8 & 261.0 & 7.1 & 273.7 & 6.6 & & & 1.4 \\
\hline & 37.9 & & & & & & & 5.2 & 267.2 & 2.6 & 304.5 & 13.9 & & & .8 \\
\hline & & & & & & & & 3 & & & & 8.9 & 25 & & .9 \\
\hline & 8.2 & & & & & & & 4 & & 10 & & 7 & & & \\
\hline & 8.2 & & & & 9 & & .7 & 4. & .0 & 5 & .1 & 4 & 274.1 & 5.9 & .9 \\
\hline & 6.1 & & & & & & .2 & 4 & & & & U & & & .9 \\
\hline 1553(I) & 267.1 & 4.2 & 241.0 & 0 & 0.2 & 7.8 & 87.4 & 3.4 & & 0.0 & 316.2 & 6.8 & & & 6.1 \\
\hline 1584(II) & & & 281.7 & 9 & & 6.9 & 277.2 & 4.0 & 299.0 & 7.2 & 283.1 & 36.3 & 3.8 & 6.1 & 1.5 \\
\hline & 0.9 & & & & & & & 4 & & 16.2 & & 27.3 & & & 3.4 \\
\hline & 1.7 & & & 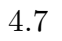 & & & & 2 & & 4 & & 4.1 & 274 & & .5 \\
\hline & 4.0 & & & 1 & & & & 3.8 & 280.2 & & & & & & .5 \\
\hline & & & & & & & .9 & 4 & & . & & & & & .4 \\
\hline (II) & 281.3 & & 270.8 & 6 & .2 & 6 & 233.2 & 6. & 209.2 & 11.5 & .0 & 3.6 & 286.1 & 5.2 & 5.2 \\
\hline (II) & 262.4 & 8.2 & 270.8 & 4.3 & 73.5 & 5.8 & 245.1 & 5.5 & 271.4 & 3.2 & 251.3 & 5.3 & 296.9 & 5.9 & 2.2 \\
\hline & 273.3 & & & U. & 285.0 & 4.1 & 299.6 & 4. & 312.0 & 5.2 & & 66 & & & 3.3 \\
\hline 1988(I) & 218.3 & 5.3 & 325.2 & 6.2 & 234.5 & 3.6 & 263.0 & 3.3 & 204.2 & 5.6 & 311.6 & 8.0 & & & 9.3 \\
\hline
\end{tabular}

Table 2 lists the complete set of radial velocities determined for the sample stars. Stars are labelled following the nomenclature of Parker (1993) and data columns include average radial velocity (and its uncertainty) for each epoch. The errors listed in columns 3 , $5,7,9,11,13$ and 15 in Table 2 correspond to the internal error $\sigma_{\text {I }}$ which is the quadratic 
sum of the standard deviation of the estimated average from the set of available lines and the minimum uncertainty $\left(3.1 \mathrm{~km} \mathrm{~s}^{-1}\right)$ as derived from high $\mathrm{S} / \mathrm{N}$ narrow nebular emission lines. Column 16 shows the ratio between the standard deviation of stellar radial velocity between different epochs and the average of $\sigma_{\mathrm{I}}$.

From the different epochs' radial velocities we can check for the presence of variations with time. To quantify this, we follow the standard procedure of comparing the dispersion of the average radial velocity for each star with the average uncertainty in the determination of each radial velocity. This can be done calculating the "external" to "internal" velocity dispersion ratio $\left(\sigma_{\mathrm{E}} / \sigma_{\mathrm{I}}\right)$ as defined by Abt et al. (1972). Radial velocity variables can then be easily flagged out as they show $\sigma_{\mathrm{E}} / \sigma_{\mathrm{I}}$ above 3 , which is similar to say that the variation in radial velocity is $3 \sigma$ above the expected uncertainties. In addition to the results presented in Table 2, the high signal to noise ratio of our spectra has also allowed us to detect several double-lined binaries that show evident variation of their absorption line profiles from epoch to epoch.

An inspection of Table 2 shows that 17 out of 46 stars show radial velocity variations. If we add the stars that present profile variations (excluding P613 which already shows radial velocity variations in Table 2 ), we have our complete set of binary star candidates, which rises to 25 out of 52 stars $(\sim 48 \%)$.

After removing the confirmed and candidate binaries, the non-binary population of our sample decreases to 26 stars, still enough to calculate a representative value of the stellar radial velocity dispersion $\left(\sigma_{\mathrm{r}}\right)$. In calculating it, we must keep in mind that each radial velocity measurement has its intrinsic error $\left(\sigma_{\text {int }}\right)$ which must be subtracted quadratically from the observed $\left(\sigma_{\mathrm{obs}}\right)$ velocity dispersion. The former is the average of the $\left\langle\sigma_{\mathrm{I}}\right\rangle$ values over the whole sample of non radial velocity variable stars while the latter is directly derived as the standard deviation around the average radial velocity derived for the same subset of stars in the cluster and it therefore follows that ${\sigma_{\mathrm{r}}}^{2}=\sigma_{\text {obs }}{ }^{2}-\left\langle\sigma_{\text {int }}\right\rangle^{2}$. For our sample, $\sigma_{\text {obs }}=10.3 \mathrm{~km} \mathrm{~s}^{-1},\left\langle\sigma_{\text {int }}\right\rangle=6.2 \mathrm{~km} \mathrm{~s}^{-1}$, which yield a value of $8.3 \mathrm{~km} \mathrm{~s}^{-1}$ for the actual radial velocity dispersion. This seems to confirm the suggestion by Bosch et al. (2001) based on simulations, that the large values derived for the stellar velocity dispersion were most probably due to the presence of binaries. As expected, if we derive the radial velocity dispersion from an individual GMOS mask observation for a single epoch, we find values as high as $30 \mathrm{~km} \mathrm{~s}^{-1}$, consistent with what was previously found in Bosch et al. (2001) from single epoch NTT data.

\section{Conclusions}

Based on six-to-seven epoch observations with GMOS on Gemini South, we have presented observational evidence that shows that the binary fraction among massive stars in NGC 2070 may be very high. Indeed we already detect a 50\% binary candidacy with only three epoch observations using medium resolution spectroscopy, which suggests that it is only a lower limit. The evidence pointing towards a high binarity fraction has an important effect on the massive end of the stellar cluster IMF, and shouldn't be overlooked.

We are aware that there are other sources for radial velocity variations among spectral features in early-type stars. However, we are confident that the variations we detected in several lines with small internal scatter are most probably due to binary nature. Regardless of the origin of the stellar radial velocity variability, the analysis of the nonvariable subset provides an important result regarding kinematics of the stellar cluster itself. The radial velocity dispersion determined for the 30 Doradus ionising cluster agrees, within observational errors, with the stellar kinematics expected if the cluster is virialised 
and its total mass is derived from the photometric plus ionised gas masses. This suggests that the stellar cluster is far from quick disruption and stands as a candidate for surviving as a future globular cluster system.

We guess that the spread among observation epochs did not allow us to derive first order orbital parameters, which would have given us important clues about period distributions. Multiple solutions with very dissimilar periods fit our current data. More observations in further epochs should allow us to confirm the binary nature of radial velocity variables detected in this work and should allow us to derive orbital parameters and individual masses of members of the binary pairs. The success of GMOS in the MOS mode makes it a very efficient tool for discovering massive binaries at large numbers using a relatively small amount of telescope time.

\section{References}

Abt, H. A., Levy, S. G., \& Gandet, T. L. 1972, AJ, 77, 138

Bosch, G. 1999, PhD thesis, Cambridge University

Bosch, G. \& Meza, A. 2001, RMxAAC, 27, 11

Bosch, G., Terlevich, R., Melnick, J., \& Selman, F. 1999, A\& AS,137, 21

Bosch, G., Selman, F., Melnick, J., \& Terlevich, R. 2001, A\&A, 380, 137

Lada, C. J. 2006, ApJ, 640, L63

Parker, J. W. 1993, AJ, 106, 560 


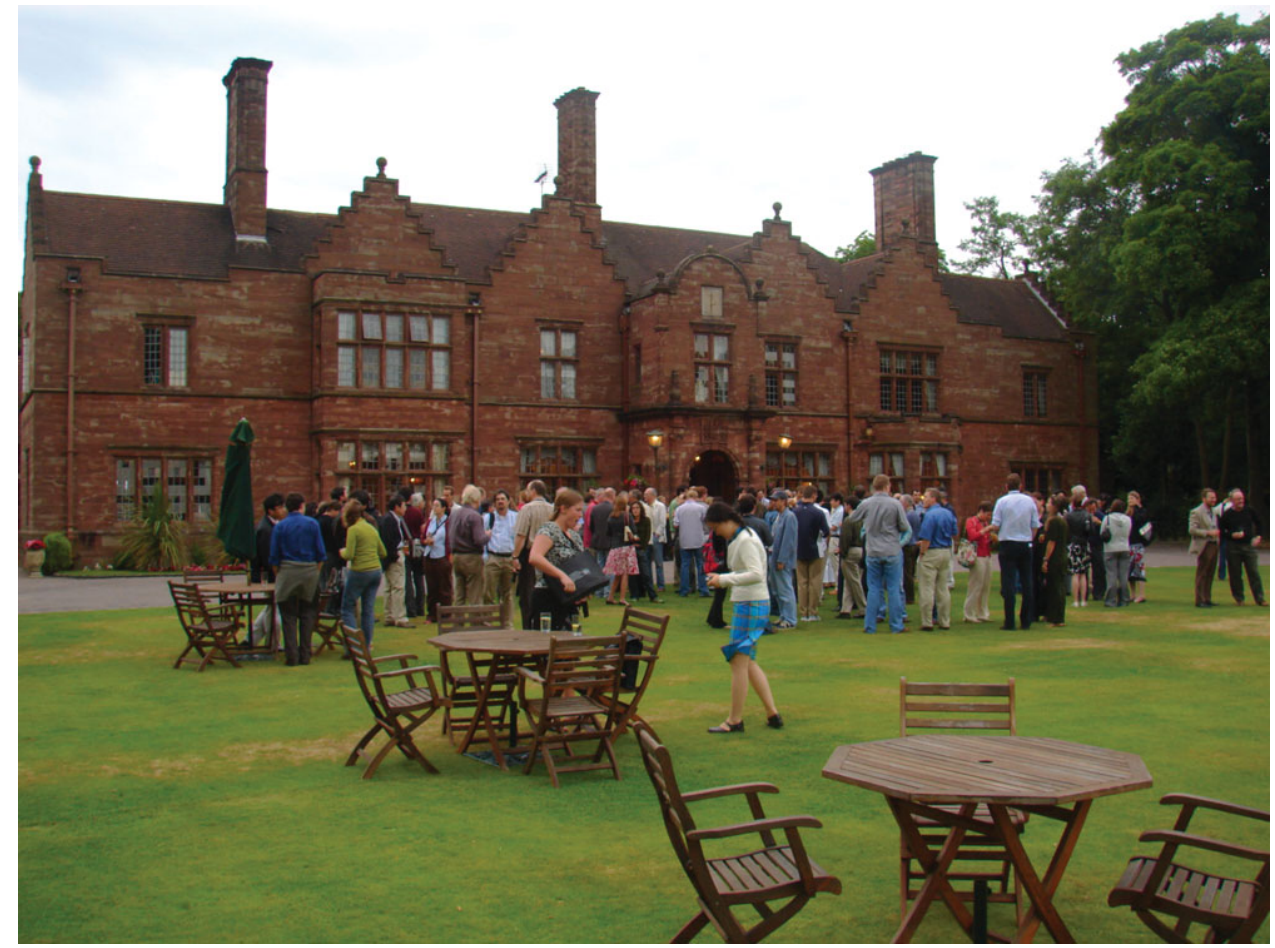

Pimms on the lawn of Wrenbury Hall.

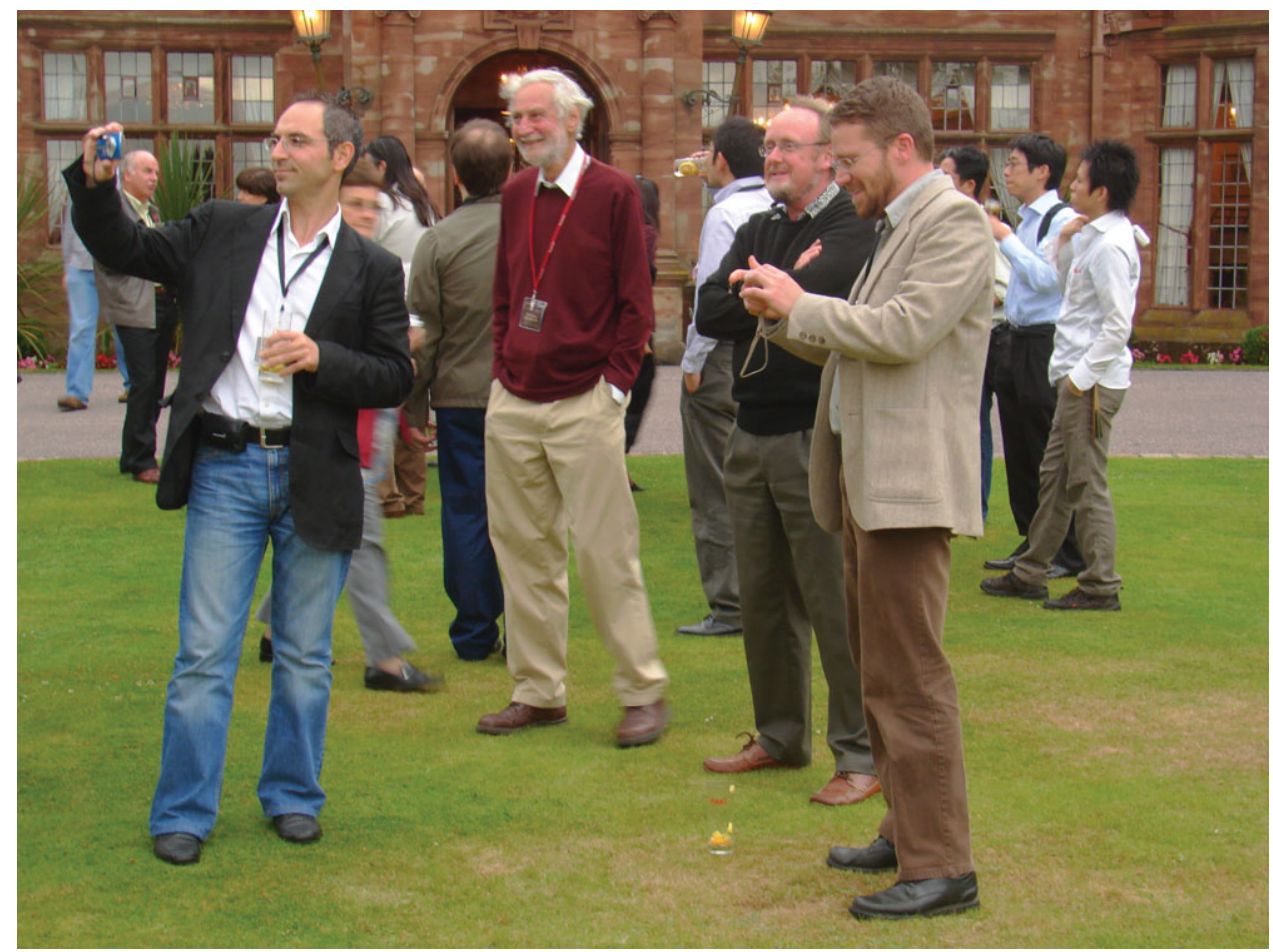

Time for pictures! 